\title{
Infecciones en huéspedes inmunocomprometidos
}

\author{
Infections in immunocompromised hosts
}

Luis Ernesto Cuéllar Ponce de León ${ }^{1}$

\section{RESUMEN}

Las infecciones son causa frecuente de morbimortalidad en huéspedes inmunocomprometidos. La frecuencia y diversidad de infecciones en esta población dependerá del tipo de inmunocompromiso, tratamiento recibido y aspecto epidemiológico. Los huéspedes con neutropenia desarrollan infecciones por bacterias extracelulares no encapsuladas y hongos levaduriformes y filamentosos; el diagnóstico precoz y tratamiento oportuno de las mismas son considerados una emergencia por su severidad y gran mortalidad asociada. El compromiso de la inmunidad humoral se relaciona con infecciones por microorganismos encapsulados (bacterias extracelulares y hongos); la secuencia clínica presentada en estas infecciones es neumonía aguda-bacteremia/fungemia-meningoencefalitis aguda/crónica. Muchas de estas infecciones son inmunoprevenibles. Los huéspedes con compromiso de la inmunidad celular desarrollan infecciones por microorganismos intracelulares y también por algunos extracelulares; muchas de las infecciones ocurren por reactivación; a menudo requieren profilaxis antimicrobiana tanto primaria como secundaria. El tratamiento medicamentoso (quimioterapia, drogas inmunosupresoras, etc.) y los procedimientos invasivos facilitan el desarrollo de determinadas infecciones. El aspecto epidemiológico, que incluye el estilo de vida, ocupación, viajes previos, etc. permite evaluar y determinar el riesgo de potenciales infecciones. En conclusión, la prevención de las infecciones y el uso racional de antimicrobianos son los pilares fundamentales en el manejo efectivo de los huéspedes inmunocomprometidos.

PALABRAS CLAVE: Infección, huésped inmunocomprometido, neutropenia (Fuente: DeCS BIREME)

\section{SUMMARY}

Infections are the most frequent causes of morbility and mortality in immunocompromised hosts. The frequency and variety of infections in this population will depend on the immunocompromised type, treatment, and epidemiological aspect. Neutropenic hosts develop infections caused by non-capsulated extracellular bacteria and levaduriform and filamentous fungi; early diagnosis and adequate treatment are extremely important to avoid high mortality in this population. Humoral immunocompromise is realated to infections produced by capsulated microorganisms (bacteria and fungi); the clinical pattern presented by this population is a progression of acute pneumonia-bacteremia/fungemia-acute/chronic meningoencephalitis. Most infections are immunopreventable. The cellular immunocompromise is related to infections produced by intracellular microorganisms and also some types of extracellular microorganisms; most of them are the result of reactivation; the use of primary and secondary antimicrobial prophylaxis is necessary; drug therapy and invasive procedures are associated with different types of microorganisms. The epidemiological aspect which includes life style, occupation, travel history, helps to assess and determine the risk of potential infections. In conclusion, preventive measures and rational use of antimicrobial drugs are critical for the effective management of immunocompromised hosts.

KEYWORDS: Infection, immunocompromised host, neutropenia (Source: MeSH NLM).

1 Médico-Cirujano. Especialista en Enfermedades Infecciosas y Tropicales. Jefe del Servicio de Infectología. Instituto Nacional de Enfermedades Neoplásicas. Coordinador del Programa VIH/SIDA/TARGA-INEN. Profesor Asociado de Medicina de la Universidad Nacional Federico Villarreal. Lima, Perú. 


\section{INTRODUCCIÓN}

El sistema inmune en el ser humano cumple actividades vitales para la conservación de la especie, entre ellas, combatir las infecciones (1) (Figura 1). La actividad anti-infecciosa se logra gracias a una extraordinaria organización del sistema inmune que controla a la gran mayoría de los microorganismos infecciosos que atacan al ser humano (2) (Figura 2).

Los huéspedes pueden ser clasificados de acuerdo al estado inmune en: inmunocompetentes e inmunocomprometidos. Existen diferentes tipos de

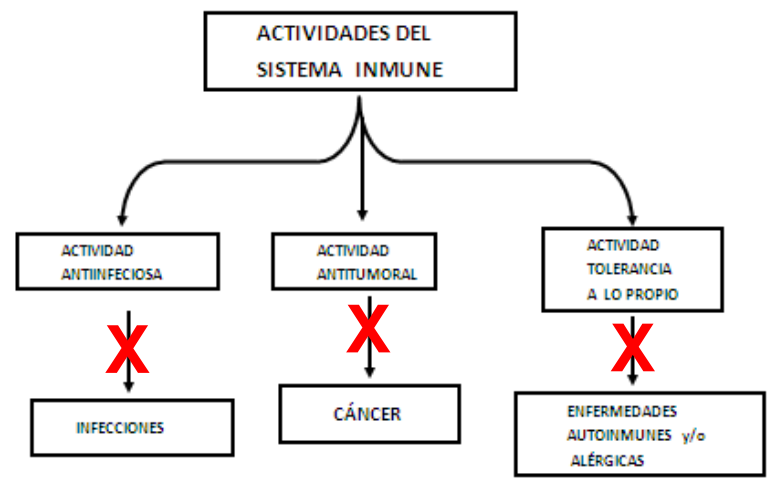

Figura 1. Actividades del Sistema Inmune. Adaptado de Kaufmamn et al (1). huéspedes inmunocomprometidos, de acuerdo al tipo de compromiso inmunitario (3-5) (Tabla 1).

Las infecciones son causas importantes de morbilidad y mortalidad en los pacientes inmunocomprometidos, siendo responsables del tipo de agentes infecciosos los siguientes factores predisponentes (6):

- El tipo de inmunocompromiso

- El tratamiento impartido

- El aspecto epidemiológico

Debido a la gran variedad y complejidad de huéspedes inmunocomprometidos, desarrollaré el tema tomando como ejemplo principalmente a los pacientes onco-hematológicos, población que permite puntualizar mejor los conceptos a presentar.

\section{Infecciones relacionadas al tipo de inmunocompromiso del huésped}

Los principales defectos inmunológicos en el ser humano son: neutropenia (deficiencia del componente fagocitario), agamma/hipogammaglobulinemia (deficiencia de la inmunidad humoral), y linfopenia T4 (deficiencia de la inmunidad celular). Cada inmunodeficiencia se relaciona con microorganismos específicos (2).

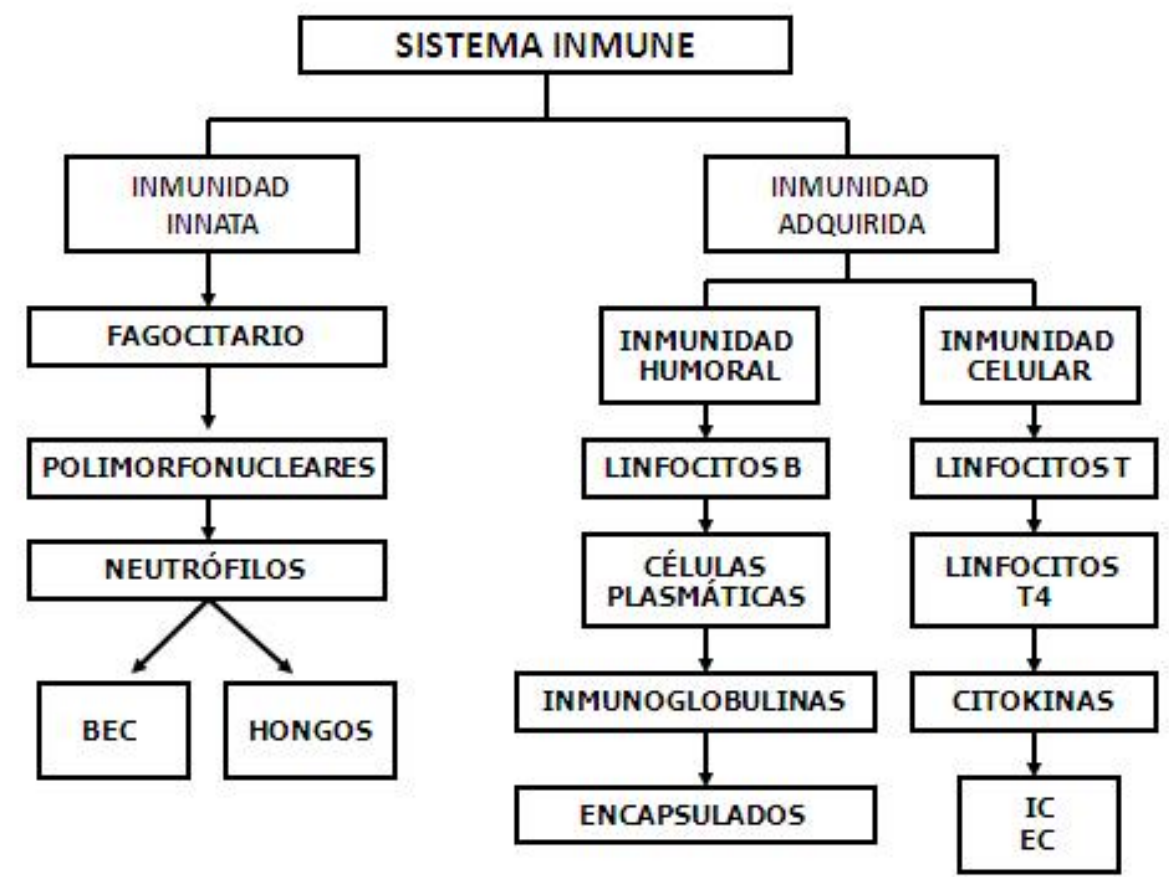

BEC: Bacterias extracelulares no encapsuladas; IC: Intracelulares; EC: Extracelulares

Figura 2. Componentes del Sistema Inmune. Adaptado de Rubin RH (2). 
Tabla 1. Principales huéspedes inmunocomprometidos.

\begin{tabular}{ll}
\hline HUÉSPEDES INMUNOCOMPROMETIDOS \\
\hline - & Neonatos \\
- & Adultos mayores \\
- & Pacientes onco-hematológicos \\
- & Transplantados (hematopoyético, órgano sólido) \\
- & Quimioterapia \\
- & Radioterapia \\
- & Enfermedad injerto-huésped \\
- & Corticoterapia crónica \\
- $\quad$ Drogas inmunosupresoras \\
- Enfermedades endocrinológicas \\
- $\quad$ Enfermedad renal crónica \\
- $\quad$ Hepatopatía crónica (cirrosis) \\
- $\quad$ Enfermedades del tejido conectivo \\
\hline
\end{tabular}

\section{Neutropenia}

Los neutrófilos son los encargados de combatir a las bacterias extracelulares no encapsuladas y a los hongos levaduriformes y filamentosos (7-9) (Tabla 2). El defecto inmunológico más importante del componente fagocitario se denomina neutropenia, que puede ser clasificada en cualitativa o cuantitativa, pero también en: neutropenia significativa $<1000$ neutrófilos $/ \mathrm{mm}^{3}$; neutropenia severa $\leq 500$ neutrófilos/ $\mathrm{mm}^{3}$; y neutropenia muy severa $\leq 100$ neutrófilos $/ \mathrm{mm}^{3}$ (7).

La neutropenia implica que la primera línea de defensa está comprometida exponiendo al huésped al desarrollo de infecciones graves por microorganismos relacionados. Las poblaciones con mayor riesgo de desarrollar neutropenia son los pacientes con leucemia aguda, en quimioterapia, en radioterapia, con anemia aplásica y en los primeros 30 días del trasplante.

En neutropenia la fiebre se define como un control de temperatura oral mayor o igual a $38,3^{\circ} \mathrm{C}$ en una toma ó 2 controles de temperatura mayor de $38^{\circ} \mathrm{C}$ en una hora (7). El riesgo de desarrollar fiebre durante un curso de quimioterapia varía entre $40 \%$ y $70 \%$. Cuando un paciente con neutropenia severa desarrolla fiebre se considera de origen infeccioso en el $60 \%$ de los casos o más; cuando se presenta en neutropenia muy severa, la infección implica bacteriemia en más del $20 \%$ de los casos; es decir, que a mayor severidad de la neutropenia las infecciones son más graves. También se considera que a mayor duración de la neutropenia, los episodios febriles son más recurrentes y más variados en microorganismos (7).

La clasificación de los episodios febriles es importante desde punto de vista epidemiológico, clínico, terapéutico y pronóstico (7) (Tabla 3). Los focos clínicos más frecuentes son el orofaríngeo, sanguíneo, pulmonar y gastrointestinal.

En el diagnóstico de neutropenia febril se deben considerar los aspectos epidemiológico, clínico y los exámenes auxiliares: cultivos, imágenes de tórax de alta resolución (TAC) y otras.

Sobre el tratamiento antimicrobiano empírico en neutropenia febril debo hacer mención lo siguiente $(6,10-16)$ :

Tabla 2. Microorganismos frecuentemente asociados a infecciones en neutropénicos.

MICROORGANISMOS
Bacterias extracelulares no encapsuladas
Bacilos gramnegativos aerobios:
Enterobacterias: E. coli, K. pneumoniae, Enterobacter sp.
No enterobacterias: Pseudomonas aeruginosa, Acinetobacter sp, S. maltophilia
Cocos grampositivos aerobios:
Staphylococcus aureus, Staphylococcus coagulasa negativo, Enterococcus faecalis y E.
faecium
Anaerobios:
Clostridium difficile, Clostridium perfringens, Clostridium septicum
Levaduriformes: Candida albicans y C. no albicans
Filamentosos: Aspergillus sp., Mucor sp.,Fusarium sp.


Tabla 3. Clasificación de los episodios febriles en neutropenia. [Adaptado de Freifeld AG. (11)].

\begin{tabular}{lc}
\hline Episodio febril en neutropenia & \\
\hline Microbiológicamente documentado & $35 \%$ \\
$\quad$ con bacteremia /fungemia & \\
$\quad$ sin bacteremia/fungemia & $22 \%$ \\
Clínicamente documentado & $43 \%$ \\
Solo fiebre (FOD en neutropenia)
\end{tabular}

- El inicio debe ser dentro de las 2 primeras horas de presentado el episodio febril. La demora del inicio, por ejemplo $24 \mathrm{~h}$ se asocia con una mortalidad mayor del $50 \%$ de los pacientes y $48 \mathrm{~h}$, con una mortalidad mayor de $80 \%$.

- La antibioticoterapia se caracteriza por ser racionalizada (empírica), de administración intravenosa, monoterapia o combinación y bactericida o fungicida (Figura 4).

Las medidas de prevención de infecciones en neutropenia son las siguientes $(6,17,18)$ :

- Precauciones estándar, principalmente higiene de manos y uso de barreras;
- Aislamiento en habitación individual con presión positiva y filtros HEPA y

- Profilaxis anti-infecciosas.

\section{Deficiencia de la inmunidad humoral}

La población de riesgo es aquella con: agammaglobulinemia o hipogammaglobulinemia congénita o adquirida, asplenia o hiposplenia, esplenectomía, Mieloma múltiple y Leucemia linfocítica crónica (19-21).

Los principales microorganismos en la deficiencia de la inmunidad humoral son las bacterias encapsuladas: neumococo, menigococo y Haemophilus influenzae, y Cryptococcus neoformans (hongo encapsulado) (19).

El cuadro clínico de la infección en huéspedes con inmunidad humoral alterada se caracteriza por la siguiente secuencia clínica: neumonía - bacteremia / fungemia - meningoencefalitis aguda / crónica.

La profilaxis antimicrobiana consiste en vacunación contra neumococo, Haemophilus influenzae, meningococo, anti influenza, y el uso de un antifúngico oral como fluconazol. La terapia antimicrobiana debe incluir alguna cefalosporina de tercera generación,

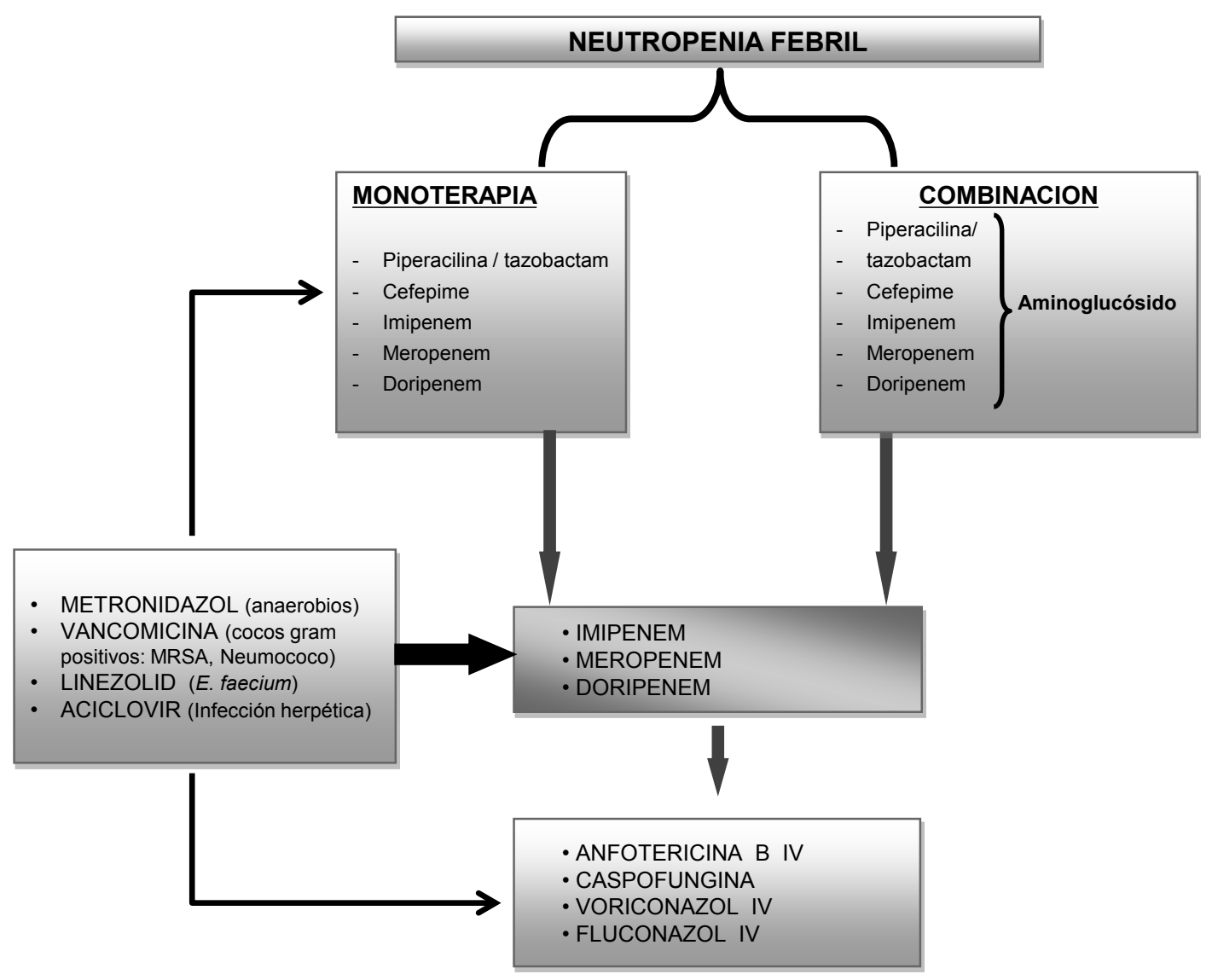

Figura 4. Terapia antimicrobiana en neutropenia febril. Adaptado de Freifeld AG (11). 
Tabla 4. Microorganismos más frecuentes en deficiencia de la inmunidad celular.

\begin{tabular}{l}
\hline Microorganismos \\
\hline Intracelulares: \\
Bacterias: Salmonella typhi y no typhi. \\
Micobacteria tb y no tb \\
Listeria monocytogenes \\
Virus: EBV,CMV,VHS 1y2, VVZ, VHB \\
Hongos: Levaduriformes \\
Endémicos \\
$\quad$ Pneumocystis jiroveci \\
$\quad$ Endoparásitos: Toxoplasma gondii \\
Extracelulares: \\
Ectoparásitos: ácaros \\
Helmintos
\end{tabular}

meropenem y antifúngicos (fluconazol y anfotericin B)(19).

\section{Deficiencia de la inmunidad celular}

La población de riesgo incluye (22-24): pacientes con Linfoma maligno Hodgkin o non Hodgkin, en corticoterapia crónica, trasplantados, con VIH/SIDA o que usan drogas inmunosupresoras.

Los microorganismos relacionados son intracelulares y extracelulares que se presentan en la tabla 4 (22). Las infecciones se desarrollan producto de reactivaciones y menos frecuentemente por infecciones "de novo" (25-33).

El cuadro clínico y la terapia antimicrobiana dependen del microorganismo involucrado y es de rutina el uso de profilaxis antimicrobiana (34).

\section{Infecciones relacionadas al tratamiento impartido}

Este factor predisponente incluye 2 tipos de elementos que sitúan a los huéspedes inmunocomprometidos en riesgo (6):

a) Medicación recibida: Quimioterapia, corticoterapia crónica, análogos de la purina, anticuerpos monoclonales, etc.

b) Procedimientos invasivos realizados: Dispositivos urinarios y vasculares, ventilación mecánica, cirugía, nutrición parenteral total, etc.

Cada uno de los elementos de riesgo se relaciona con determinado tipo de microorganismos infecciosos.

\section{Infecciones relacionadas con la epidemiología}

Incluye tener en cuenta lugar de nacimiento del huésped y de los padres, lugar de procedencia, ocupación, estilo de vida, etc $(2,30)$.

\section{CONCLUSIONES}

Luego de la revisión se puede concluir que existen múltiples tipos de huéspedes inmunocomprometidos y una gran variedad de microorganismos que se relacionan específicamente con cada uno de los defectos inmunitarios. Por esto es importante realizar un adecuado enfoque clínico ante un huésped inmunocomprometido febril y/o con un foco clínico.

Además se debe mencionar que los huéspedes pueden ser portadores de deficiencias inmunitarias de aparición simultánea o en el tiempo, por ejemplo, un huésped con mieloma múltiple presenta de base deficiencia en la inmunidad humoral, al ser sometido a quimioterapia, desarrolla neutropenia y si es sometido a trasplante la inmunidad celular se compromete.

Finalmente, las estrategias de prevención de infecciones más importantes a practicar en esta población son: medidas de control y prevención de infecciones (precauciones estándard) y una política de uso racional de antimicrobianos (profilaxis y tratamiento).

\section{Agradecimiento:}

Al Sr. Luis Paul Condorí Sotomayor por el apoyo en la elaboración del artículo.

\section{Correspondencia}

Luis Ernesto Cuéllar Ponce de León

Correo electrónico: 1cuellar@inen.sld.pe

\section{REFERENCIAS BIBLIOGRÁFICAS}

1. Kaufmann SH, Sher A, Ahmed R, editors. Inmunology of infectious diseases. Washington DC: ASM Press; 2002.

2. Rubin RH, Young LS, editors. Clinical approach to infection in the compromised host. $4^{\text {th }}$ ed. New York: Kluwer Academic/Plenium Publishers; 2002.

3. Glauser MP, Pizzo PA. Management of infections in immunocompromised patients. United Kingdom: WB Saunders Co.; 2000.

4. Aguado JM. Infecciones en pacientes transplantados. Madrid: Harcour S.A.;2000. 
5. Bowden RA, Lungman P, Paya CV, editors. Transplant infections. $3^{\text {rd }}$ ed. Philadelphia: Lippincott Williams \& Wilkens; 2010.

6. Cuéllar L. Infecciones en Inmunocomprometidos. Rev Per Enferm Infecc Trop. 2006; 5(1):17-18.

7. Rolston KV, Rubenstein EB, editors. Textbook of febrile neutropenia. London: Martin Dunitz Ltd; 2001.

8. Díaz-Mediavilla J, Lizasoain M. Epidemiología de las infecciones en el paciente neutropénico. Enferm Infecc Microbiol Clin. 2005; 23(S5):7-13.

9. Padrón N, Gra S. Infecciones en el paciente neutropénico con cáncer. Rev Panam Infectol. 2006; 8 (3):24-34.

10. Jarque J, Salavert M, Sanz MA. Manejo del paciente neutropénico con fiebre. Enferm Infecc Microbiol Clin. 2005; 23(S5): 24-29.

11. Freifeld AG, Bow EJ, SepkowitzKA et al. Clinical practice guideline for the use of antimicrobial agents in neutropenic patients with cancer: 2010 Update by the Infectious Diseases Society of America. Clin Infect Dis. 2011; 52(4):e56-e93.

12. Klastersky J, Awada A, Paesmans M, Aoun M. Febrile neutropenia: A critical review of the initial management. Crit Rev Oncol Hematol. 2011; 78(3):185-194.

13. Bal AM, Gould IM. Empirical antimicrobial treatment for chemotherapy-induced febrile neutropenia. Inter J Antimicrob Agents. 2007; 29:501-509.

14. Goldberg E, Gafter-Gvili A, Robenshtok E,Leibovici L,Paul M. Empirical antifungal therapy for patients with neutropenia and persistent fever: systematic review and meta-analysis. Eur J Cancer. 2008; 44: 2192-2203.

15. Vallejo JC, Ruiz I. Infección fúngica invasora en los pacientes hematológicos. Enferm infecc Microbiol Clin. 2012; 30(9):572-579.

16. Viscoli C, Varnier O, Machetti M. Infections in patients with febrile neutropenia: epidemiology, microbiology and risk stratification. Clin Infect Dis. 2005; 40:S2405.

17. Uriburu C, Rovira M. Profilaxis de las infecciones en el paciente neutropénico. Enferm Infecc Microbiol Clin. 2005; 23(S5):14-18.

18. Flowers CR, Seidenfeld J, Bow EJ, et al. Antimicrobial prophylaxis and outpatient management of Fever and neutropenia in adults treated for malignancy: american society of clinical oncology clinical practice guideline. J Clin Oncol. 2013; 31(6):794-810.

19. Davies JM, Lewis MPN, Wimperis J, et al. Review of guidelines for the prevention and treatment of infection in patients with an absent or dysfunctional spleen: prepared on behalf of the British Committee for Standards in Haematology by a working party of the Haemato-Oncology task force. Br J Haematol. 2011; 155:308-317.
20. Cadili A, de Gara C. Complications of splenectomy. Am J Med. 2008; 121:371-375.

21. Nucci M, Anaissie E. Infections in patients with Multiple Myeloma. Semin Hematol 2009; 46: $277-$ 288.

22. Rovira M, Ruiz I. Infecciones en el transplantado de progenitores hematopoyéticos. Enferm Infecc Microbiol Clin. 2007; 25(7):477-86.

23. Pérez JL, Ayats J, Fortún J,Pumarola T. Microbiología del trasplante. Enferm Infecc Microbiol Clin. 2011; 29(9):683-690.

24. Martín-Dávila P, Blanes M, Fortún J. Inmunosupresión e infección en el paciente trasplantado. Enferm Infecc Microbiol Clin. 2007; 25(2):143-54.

25. Gavaldá J, Meije Y, Len O, Pahissa A. Infección fúngica invasora en el transplantado de órgano sólido. Enferm Infecc Microbiol Clin. 2012; 30(10):645653.

26. Tamura A, Hebisawa A, Tanaka G, et al. Active pulmonary tuberculosis in patients with lung cáncer. Kekkakku 1999; 74:797-802.

27. Cuéllar L, Biminchumo C, Rosales R, Vicente W, Pinto J, Miranda L. Tuberculosis activa en pacientes con cáncer: experiencia del INEN: 1995-2004.Lima, Perú: X Congreso Peruano de Enfermedades Infecciosas y Tropicales; 2007.

28. Ravandi F, O'Brien S. Infections associated with purine analogs and monoclonal antibodies. Blood Rev. 2005; 19:253-273.

29. Cuéllar L. Bartonelosis en una paciente con enfermedad de Hodgkin. Rev Per Enferm Infecc Trop. 2001; 1(3):139-140.

30. Khardori N, Khardori R, guest editors. Preface (Internet). Infectious Disease Clinics of North America. 2007; 21(3). (Citado en febrero del 2013) Disponible en: http://www.id.theclinics.com/article/ S0891-5520(07)00067-0/fulltext

31. Nacci F, Matucci-Cerinic M. Tuberculosis and other infections in the antitumour necrosis factor-alpha (anti-TNF alpha) era. Best Pract Res Clin Rheumatol 2011;25: 375-388.

32. Marr KA, Subramanian AK, guest editors. Infections in transplant and oncology patients (Internet). Infect Dis Clin North Am. 2010; 24(2):13-15. (Citado en febrero del 2013) Disponible en: http:/www. id.theclinics.com/article/S0891-5520(10)00016-4/ fulltext

33. Hayden RT, Carroll KC, Tang YW, Wolk DM, editors. Diagnostic microbiology of the immunocompromised host. Washington DC: ASM Press; 2009.

Recibido: 28/02/2013

Aceptado: 08/05/2013 